\title{
Effect of Combined Therapy Using Diode Laser and Photodynamic Therapy on Levels of IL-17 in Gingival Crevicular Fluid in Patients With Chronic Periodontitis
}

\author{
Abhishek Mistry", Richard Pereira, Vineet Kini, Ashvini Padhye \\ Department of Periodontics, Mahatma Gandhi Mission's Dental College and Hospital, Junction of NH4 and Sion-Panvel \\ Expressway, Kamothe, Navi Mumbai- 410209, India
}

\author{
*Correspondence to \\ Abhishek Mistry, MDS; Department \\ of Periodontics, Mahatma Gandhi \\ Mission's Dental College and \\ Hospital, Junction of NH4 and Sion- \\ Panvel Expressway, Kamothe, Navi \\ Mumbai-410209. \\ Tel: +91 9773320697; \\ Email: abhishek1561@gmail.com
}

Published online 27 October 2016

\section{Introduction}

Periodontal therapy aims at removal of hard and soft deposits from the root surface. ${ }^{1}$ Hand and ultrasonic instrumentation for scaling and root planing (SRP) have shown to be effective approaches for nonsurgical periodontal therapy. ${ }^{2}$ Lasers have been suggested as a new alternative for treatment of periodontal disease. ${ }^{3}$ Diode laser, neodymium-doped: yttrium, aluminum and garnet laser (Nd:YAG), erbium-doped: yttrium, aluminum and garnet laser (Er:YAG) and carbon dioxide (CO2) laser ${ }^{4}$ with wavelengths $(\lambda)$ ranging from $630 \mathrm{~nm}$ to $10600 \mathrm{~nm}$ are commonly used for periodontal therapy. Lasers have been considerably used due to their antibacterial potential by direct ablation, thermal denaturation, and destruction of bacterial cells.

Photodynamic therapy (PDT) combines the use of visible light and a photo sensitizer, to produce singlet oxygen molecules and free radicals which have an antimicrobial effect. ${ }^{5}$ The use of helium/neon $(\mathrm{He} / \mathrm{Ne})$ laser or gallium-aluminium arsenide (GaAlAs) laser with a suitable photo sensitizer showed a significant reduction of bacteria in subgingival plaque from subjects with chronic periodontitis. ${ }^{6}$ Bacteria associated with periodontal dis- ease can also be killed through photosensitization using toluidine blue $\mathrm{O}$ with $\mathrm{He} / \mathrm{Ne}$ laser. ${ }^{7}$ A positive effect on pocket depth reduction as well as improvement in clinical levels and bleeding on probing (BOP) have also been reported. ${ }^{8,9}$ Lui et al showed a beneficial effect when they combined photodynamic and low level laser therapy in nonsurgical treatment of chronic periodontitis. ${ }^{10}$

Periodontitis is a disease with multi factorial etiology causing the destruction of supporting structures resulting in formation of periodontal pocket and loss of alveolar bone. ${ }^{11}$ The major determinant of this disease susceptibility is the host immune inflammatory response to the subgingival biofilm. The pathogenesis of periodontitis involves role play of cytokines, produced by host defense cells in reaction to antigenic stimuli, which have a wide range of overlapping functions. These cytokines are soluble proteins that paradoxically result in maximum tissue damage leading to the clinical manifestation of the disease.

Interleukin-17 (IL-17) is one such pro-inflammatory cytokine that is involved in causing loss of alveolar bone in periodontitis. ${ }^{12}$ This cytokine is produced by activated $\mathrm{CD} 4+\mathrm{T}$ cells $^{13,14}$ and neutrophils. ${ }^{15,16}$ IL-17 expression

Please cite this article as follows: Mistry A, Pereira R, Kini V, Padhye A. Effect of combined therapy using diode laser and photodynamic therapy on levels of IL-17 in gingival crevicular fluid in patients with chronic periodontitis. J Lasers Med Sci. 2016;7(4):250-255. doi:10.15171/jlms.2016.44. 
was reported to be increased in gingivitis. ${ }^{17}$ Its expression was also reported to be increased in gingival crevicular fluid (GCF) obtained from sites with periodontitis. ${ }^{18} \mathrm{GCF}$ levels of IL-17 significantly reduced following nonsurgical periodontal therapy. ${ }^{19,20}$ Thus IL-17 serves as a useful marker indicative of disease states. ${ }^{21}$

As limited data exist on combined therapy of laser and PDT in the treatment of patients with periodontitis, this study aims to compare the efficacy of combination therapy of diode laser and PDT as an adjunct to SRP in the treatment of chronic periodontitis and use the above described IL-17 marker as a diagnostic indicator of disease regression.

\section{Methods}

Subjects

Thirty Indian adults (19 males and 11 females with a mean age of 38.03 years) were recruited from the Mahatma Gandhi Mission's Dental College and Hospital, Navi Mumbai. Inclusion criteria were (i) subjects aged 30-49 years, (ii) dentition with at least 20 functional teeth, and (iii) subjects with chronic periodontitis having more than two nonadjacent sites with probing pocket depth (PPD) $\geq 5 \mathrm{~mm}$, interproximal attachment loss of $\geq 3 \mathrm{~mm}$ and radiographic evidence of alveolar bone loss. Exclusion criteria were $(i)$ subjects with diabetes mellitus, rheumatoid arthritis, irritable bowel disease, pulmonary disease, multiple sclerosis, bacterial, viral and fungal infections, (ii) subjects who have received any form of periodontal therapy within the past 6 months, (iii) subjects with aggressive periodontitis, (iv) subjects who have received antibiotic therapy within the past 6 months, (v) subjects on anti-inflammatory drug regimen during the past 6 months, ( $v i)$ the presence of pulpal or periapical involvement on qualifying teeth, (vii) subjects who have received orthodontic treatment in the past 6 months, (viii) pregnant and lactating women, $(i x)$ physically and mentally challenged subjects, and $(x)$ Smokers. All subjects were informed about the study and gave their consent for the study.

Study Design and Periodontal Examination

The present study was a split mouth double blinded, prospective cohort, and randomized controlled clinical trial.
An experienced clinician (MR) who was blinded to the study procedure collected all clinical data at the baseline and during follow-up visits while another experienced periodontist carried out the desired treatment procedures. Ten patients with probing depth (PD) $>5 \mathrm{~mm}$ on $\geq 1$ aspect of each tooth, who were not included in the study were recruited for calibration of the main examiner (MR). The examiner recorded full-mouth PDs at six sites per tooth (excluding the third molars) in two different sessions on these subjects, using a UNC-15 probe. Upon completion of all measurements, the intraexaminer repeatability for PD measurement was assessed. Calibration was accepted if the measured parameters at two appointments were similar at a $>90 \%$ level. At the initial visit all subjects underwent full-mouth periodontal examination and based on the inclusion criteria, teeth were randomly allocated to the test group (SRP plus combined therapy using diode laser and PDT) or control group (SRP alone). The subjects then received one stage full mouth SRP under local anesthesia (2\% lignocaine with 1:80000 adrenaline) using ultrasonic scaler (Satelec, Suprasson Newron, France) and/or Gracey curettes (Hu-Friedy \#1-14, USA) and routine oral hygiene instructions. Full mouth alginate impressions were made and poured in dental stone to make the diagnostic cast on which customized acrylic stents was fabricated. These stents were used to assure reproducible measuring points for $\mathrm{PPD}$ and relative attachment levels (RALs). The patients were recalled after 1 week for collection of GCF to assess the levels of IL-17, which was recorded as our baseline data. Clinical parameters namely PD, RAL, BOP and gingival index (GI) were also recorded. After removal of supragingival plaque, therapy using diode laser and PDT was performed for the test teeth. GCF samples were again collected after 3 months to assess the levels of IL-17. Clinical parameters were recorded at 1 month and 3 months respectively (Figure 1).

Gingival Crevicular Fluid Sampling and Assay Supragingival plaque was removed and teeth were isolated using cotton rolls, microcapillary pipette was used for collection of GCF by placing it extra crevicular at the entrance of the gingival sulcus. Blood-contaminated sam-

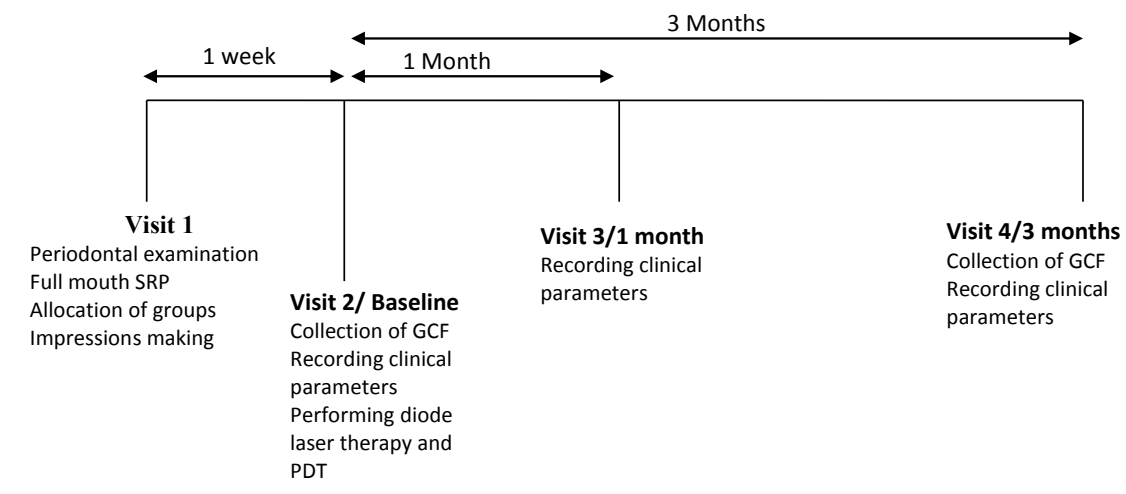

Figure 1. Study Design. Abbreviations: SRP, scaling and root planning; GCF, gingival crevicular fluid; PDT, photodynamic therapy. 
ples were discarded. Five microliters of GCF was collected from each site, placed in $100 \mu \mathrm{L}$ of sterile phosphate buffered saline ( $\mathrm{pH}$ 7.2) in Eppendorf tubes. The samples were stored at $-70^{\circ} \mathrm{C}$. IL- 17 was analyzed using an ELISA kit (Human IL-17A (homodimer) ELISA Ready-SETGo! ${ }^{\circledR}$ From eBioscience), and values were presented as $\mathrm{pg} / \mu \mathrm{L}$.

\section{Recording of Clinical Parameters}

PD was measured at six sites per tooth i.e., mesiobuccal, midbuccal, distobuccal, mesiolingual, midlingual and distolingual, from the gingival margin to the base of the gingival pocket using a UNC-15 probe (Hu-Friedy, USA). RAL was measured as the distance from a fixed reference point (FRP) on the customized acrylic stent to the base of the pocket with a UNC-15 probe.

BOP was recorded using Ainamo and Bay index (1975) and GI by Loe and Silness (1963).

\section{Adjunctive Laser Treatments}

After collection of GCF and recording of clinical parameters, the test teeth underwent therapy using diode laser and PDT. Topical anesthetic gel (2\% lignocaine Hydrochloride, Neon Laboratories Limited, India) was applied around the concerned teeth and then washed off after 5 minutes with the help of sterile saline. The test teeth first underwent laser therapy using $810 \mathrm{~nm}$ diode laser (The Picasso diode laser, AMD Lasers, USA). The laser beam was delivered into the periodontal pocket using a $400 \mu \mathrm{m}$ fiber optic tip in contact mode from apical to coronal direction using a sweeping motion for 1 minute using $1 \mathrm{~W}$ as a continuous wave. ${ }^{22}$ Thermal injury was minimized by keeping the tip in constant motion throughout the procedure. Also, diode laser $(\lambda=810 \mathrm{~nm})$ has a low tissue penetration allowing complete removal of the gingival epithelium with minimal damage to lamina propria. ${ }^{23}$

Following which, the same teeth underwent PDT. The periodontal pocket was flooded with $2 \mathrm{~mL}$ of $1 \%$ methylene blue (S D Fine-Chem Limited, India) from the apical to coronal end of the pocket with the help of a blunt cannula. Three minutes later, the pocket was thoroughly rinsed with saline to remove excess photosensitizer. Following this, an $810 \mathrm{~nm}$ diode laser was operated at 0.1 $\mathrm{W}$ using a $400 \mu \mathrm{m}$ fiber optic tip as a continuous wave. The tip was introduced in contact mode at the base of the periodontal pocket from where it was moved in coronal direction using sweeping motion for 1 minute followed by additional vertical movements in apical and coronal directions for 30 seconds. The laser therapy was performed by an experienced periodontist who was not involved in the periodontal examination and data collection. The patient, operator, and dental assistant used safety glasses for protection of the eyes from the laser beam. The laser therapy was carried out in a closed operatory room, with a warning sign at the door to inform those outside.

Statistical Analysis

The distributions of the parameters were checked for nor- mality using the one-sample Kolmogorov-Smirnov test. Because not all data were normally distributed, a decision was taken to use non-parametric tests. To test the mean equality of the two groups with respect to IL-17 level in GCF and the clinical parameters, Kruskal-Wallis was carried out at $5 \%$ level of significance. To compare the changes in the IL-17 level in GCF, PD, RAL, BOP, GI at 3 time intervals within test and control groups, Wilcoxon signed rank test was performed. Pair wise comparison of groups at various time intervals was done using Mann-Whitney $\mathrm{U}$ test. Differences were considered as statistically significant at $P<0.05$. The statistical analysis was done using SPSS software (SPSS for Windows, release 16.0; SPSS Inc., Chicago, IL, USA).

\section{Results}

Thirty patients (19 males and 11 females, mean age 38.03) participated in the study. There were no dropouts in the study. Overall, 60 sites (30 test and 30 control) were evaluated for IL-17 levels in GCF.

The results for IL-17levels in GCF for both groups at baseline and 3 months are presented in Table 1 and Figure 2. A significant reduction in IL-17 level was seen in both test and control groups 3 months after the treatment $(P<0.05)$. However, when comparing both groups no statistically significant difference was seen.

The PPD and RAL results (mean \pm SD) for both groups at baseline, 1 month and 3 months are presented in Table 2 . Throughout the study a significant reduction of the PD and a significant gain of RAL took place in both groups $(P<0.05$; Table 2, Figures 3 and 4$)$. However, no statistical significant difference was seen when comparing both groups at 3 time intervals i.e., baseline, 1 month and 3

Table 1. IL-17 Level in GCF: Mean Scores ( \pm SD; $N=30$ patients) at Baseline and 3 Months

\begin{tabular}{llll}
\hline Treatment & Baseline $( \pm$ SD) & 3 Months $(\underline{\underline{S} \text { SD })}$ & $\boldsymbol{P}$ Value \\
\hline IL-17 levels & & & \\
Test teeth & $687.12 \pm 714.37$ & $244.14 \pm 205.48$ & $*$ \\
Control teeth & $576.19 \pm 509.32$ & $186.58 \pm 107.94$ & $*$ \\
$P$ value & NS & NS & \\
$* P<0.05, N S=$ not significant. &
\end{tabular}

$* P<0.05, \mathrm{NS}=$ not significant.

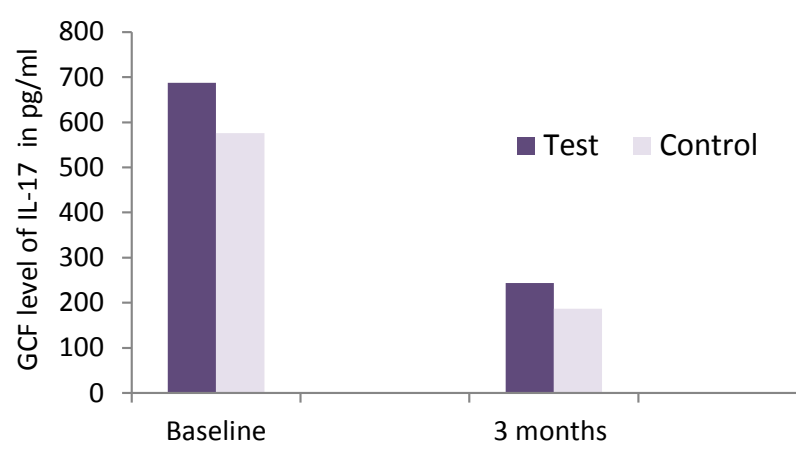

Figure 2. Comparison of Level IL-17 in GCF Over 3-Month Time Period. 
Table 2. PPD and RAL: Mean Scores ( \pm SD; $N=30$ Patients) at Baseline, 1 Month and 3 Months

\begin{tabular}{llll}
\hline Treatment & Baseline $(\underline{\underline{\mathbf{S D}})}$ & 1 Month $( \pm \mathbf{S D})$ & 3 Months $( \pm$ SD) \\
\hline PPD & & & $\boldsymbol{P}$ Value \\
Test teeth & $5.03 \pm 0.183$ & $3.27 \pm 0.450$ & $3.07 \pm 0.521$ \\
Control teeth & $5.13 \pm 0.346$ & $3.27 \pm 0.450$ & $2.97 \pm 0.490$ \\
$P$ value & $\mathrm{NS}$ & $\mathrm{NS}$ & $\mathrm{NS}$ \\
RAL & & $7.13 \pm 1.137$ & $6.93 \pm 1.112$ \\
Test teeth & $8.90 \pm 0.960$ & $7.07 \pm 1.048$ & $6.77 \pm 1.104$ \\
Control teeth & $8.97 \pm 1.129$ & $\mathrm{NS}$ & $\mathrm{NS}$ \\
$P$ value & $\mathrm{NS}$ & $*$ \\
\hline
\end{tabular}

Abbreviations: NS, not significant; PPD, probing pocket depth; RAI, relative attachment level. $* P<0.05$.

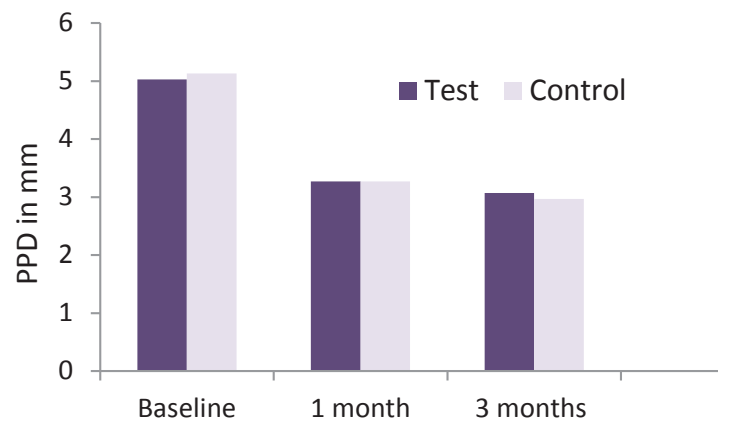

Figure 3. Comparison of PPD Over the 3-Month Time Period.

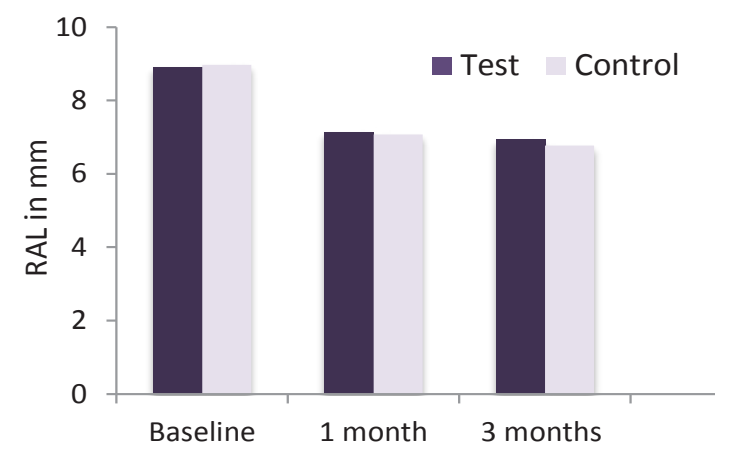

Figure 4. Comparison of RAL Over the 3-Month Time Period.

months (Table 2).

The BOP and GI results (mean \pm SD) for both groups at baseline, 1 month and 3 months are presented in Table 3, Figures 5, and 6. A significant reduction in BOP and the GI was seen in both groups after 1 month and 3 months of therapy. However the best reduction in BOP and GI was seen in test group as compared to control group and it reached statistical significance $(P<0.05$; Table 3$)$.

\section{Discussion}

In the present study, we used combination therapy of diode laser and PDT as an adjunct to SRP.

Diode laser helps to reduce subgingival microbiota thus providing sites for new connective tissue attachment. ${ }^{24}$ It also helps in the promotion of hemostasis, has detoxifying effects and enhances healing. In addition, PDT using methylene blue dye exerts antimicrobial effect, by

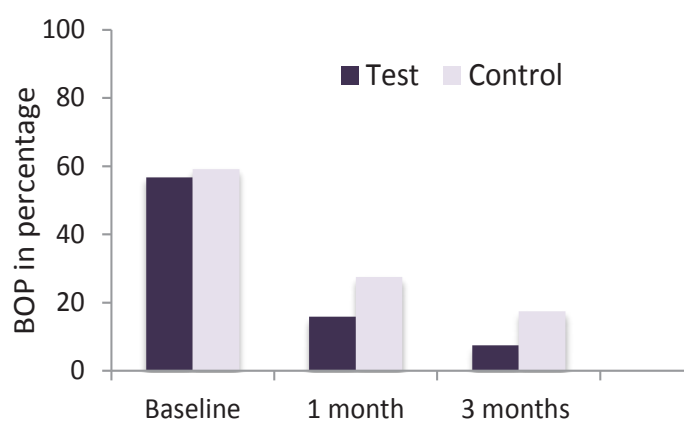

Figure 5. Comparison of BOP Over the 3-Month Time Period.

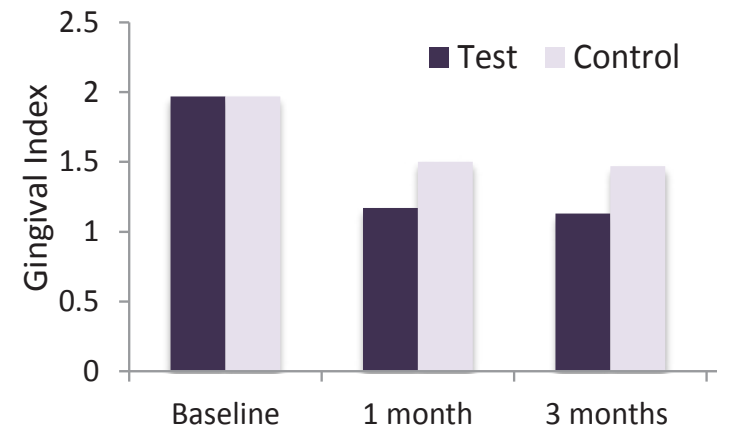

Figure 6. Comparison of Gl Over the 3-Month Time Period.

allowing methylene blue to enter gram-negative bacteria through the porin-protein channels of the outer membrane which upon activation using diode laser, releases oxidizing metabolites that have harmful effects on lipopolysaccharide. $^{25}$

Th1/Th2 paradigm has an important role in the host immune defense responses in chronic periodontitis. ${ }^{26}$ However with the discovery of Th17 cells and Th17-related cytokines, pathogenesis of periodontitis has seen a new trend. We analyzed GCF levels of IL-17, at baseline and 3 months after the completion of treatment. IL-17 is a pro-inflammatory cytokine that is involved in causing an alveolar bone loss in periodontitis. Vernal et al reported high levels of IL-17 in GCF and in the culture supernatants of gingival cells of patients with periodontitis. ${ }^{18}$ Takahashi et al also suggested an important role of IL17 in the pathogenesis of periodontal disease. ${ }^{21}$ We also 
Table 3. BOP and GI: Mean Scores ( \pm SD; $N=30$ Patients) at Baseline, 1 Month and 3 Months

\begin{tabular}{|c|c|c|c|c|}
\hline Treatment & Baseline $( \pm \mathrm{SD})$ & 1 Month ( \pm SD) & 3 Months $( \pm$ SD) & $P$ Value \\
\hline \multicolumn{5}{|l|}{$\mathrm{BOP}$} \\
\hline Test teeth & $56.67 \pm 22.68$ & $15.83 \pm 16.72$ & $7.50 \pm 14.90$ & $*$ \\
\hline Control teeth & $59.17 \pm 55.25$ & $27.50 \pm 15.19$ & $17.50 \pm 16.29$ & * \\
\hline$P$ value & NS & * & $*$ & \\
\hline \multicolumn{5}{|l|}{ GI } \\
\hline Test teeth & $1.97 \pm 0.183$ & $1.17 \pm 0.379$ & $1.13 \pm 0.346$ & $*$ \\
\hline Control teeth & $1.97 \pm 0.183$ & $1.50 \pm 0.509$ & $1.47 \pm 0.507$ & $*$ \\
\hline$P$ value & NS & $*$ & $*$ & \\
\hline
\end{tabular}

Abbreviations: NS, not significant; GI, gingival index; BOP, bleeding on probing.

$* P<0.05$.

reported a significant increase in levels of IL-17 in GCF in patients with chronic periodontitis. On the contrary a study by Pradeep et al found no significant relationship between IL-17 and periodontitis. ${ }^{27}$ In our study, we found a statistical significant reductions in levels of IL17 in both treatment groups 3 months after treatment, however when comparing both treatment modalities no significant difference was found. This change in levels of IL-17 after treatment is in accordance with various other studies which also reported significant reductions in levels of IL-17 following treatment. Giannopoulou et al found statistically significant reductions in levels of IL-17 after nonsurgical periodontal therapy with SRP, lasers and PDT. $^{22}$ Zhao et al.also found statistically significant reductions in levels of IL-17 after SRP. ${ }^{19}$ Shirmohammadi et al also found statistically significant reductions in levels of IL-17 after one stage full mouth disinfection and SRP. ${ }^{20}$ Our study results also showed statistically significant reductions in PDs and RALs in both test and control groups at 1 month and 3 months intervals. When comparing the test and control groups, no statistically significant results were found in respect to PDs reductions and RALs gains. This is in accordance with various studies where no statistically significant difference in reduction in PDs and gain in attachment levels were seen in the test group when compared to control groups after an additional treatment using PDT. ${ }^{10,28-31}$ However our results are not in keeping with a number of recent clinical investigations, which yielded significantly better clinical results for SRP in conjunction with adjunctive PDT than for SRP alone. ${ }^{8,9,32}$

Test teeth showed a significantly greater reduction in the percentage of sites with BOP compared with control teeth at 1 month and 3 months after treatment. Similar results were obtained by Lui et al when they combined low level laser therapy and PDT. ${ }^{10}$ This is in accordance with various other studies which also reported significant greater reductions in BOP after adjunctive use of $\mathrm{PDT}^{9,29,30,32}$ Moritz et al showed a significant reduction in bacteria and inflammation when he combined diode laser therapy with SRP. ${ }^{24}$ This is in contrast to various other studies which failed to note significant greater improvements after adjunctive use of PDT. ${ }^{22,28}$ Another notable effect was the statistically significant reduction in GI in the test group after one month and three months. Similar changes in GI were reported by de Oliveira et al and Yilmaz et al after PDT as compared to SRP. ${ }^{31,33}$
On the basis of our results, we suggest that reduction in IL-17 levels of GCF after combined therapy using diode laser and PDT or SRP corresponds to the clinical improvements seen in the periodontal status and it can be used as a potential marker of periodontal disease activity. Further use of combined therapy with diode laser and PDT may provide additional benefit as they may help in reduction of key virulence factors such as lipopolysaccharide and proteases. ${ }^{34}$ However, at present there is no established protocol for use of lasers as an adjunct to SRP. Therefore, more studies are required to establish an effective treatment protocol of the combination of this treatment modality in the treatment of patients with chronic periodontitis.

\section{Conclusion}

The results show that, both treatment modalities resulted in significant decrease in level of IL-17 in GCF in patients with chronic periodontitis suggesting the efficacy of combination therapy of diode laser and PDT as an adjunct to SRP on reducing levels of IL-17 in GCF in patients with chronic periodontitis.

\section{Ethical Consideration}

The study was conducted in full accordance with the declared ethical principles (World Medical Association Declaration of Helsinki, version VI, 2002) and had been approved by the local Ethical Committee (Reference number: MGM/DCH/IEC/11/2012).

\section{Conflict of Interests}

Authors have no conflict of interest to declare.

\section{References}

1. Cobb CM. Non-surgical pocket therapy: Mechanical. Ann Periodontol. 1996;1(1):443-490.

2. Van der Weijden GA, Timmerman MF. A systematic review on the clinical efficacy of subgingival debridement in the treatment of chronic periodontitis. J Clin Periodontol. 2002;29 Suppl 3:55-71. doi:10.1034/j.1600-051x.29.s3.3.x.

3. Aoki A, Sasaki KM, Watanabe H, Ishikawa I. Lasers in nonsurgical periodontal therapy. Periodontol 2000. 2004;36:59-97. doi:10.1111/j.1600-0757.2004.03679.x.

4. Schwarz F, Aoki A, Becker J, Sculean A. Laser application in non-surgical periodontal therapy: a systematic review. J Clin Periodontol. 2008;35(8 Suppl):29-44. doi:10.1111/ j.1600-051x.2008.01259.x. 
5. Konopka K, Goslinski T. Photodynamic therapy in dentistry. $J$ Dent Res. 2007;86(8):694-707. doi:10.1177/154405910708600803.

6. Wilson M, Burns T, Pratten J, Pearson GI. Bacteria in supragingival plaque samples can be killed by low-power laser light in presence of a photosensitizer. J Appl Bacteriol. 1995;78(5):569-574.

7. Dobson J, Wilson M. Sensitization of oral bacteria in biofilms to killing by light from a low-power laser. Arch Oral Biol. 1992;37(11): 883-887.

8. Andersen R, Loebel N, Hammond D, Wilson M. Treatment of periodontal disease by photodisinfection compared to scaling and root planing. J Clin Dent. 2007;18(2):34-48.

9. Braun A, Dehn C, Krause F, Jepsen S. Short term clinical effects of adjunctive antimicrobial photodynamic therapy in periodontal treatment; a randomized clinical trial. J Clin Periodontol. 2008;35(10): 877-884. doi:10.1111/j.1600051x.2008.01303.x.

10. Lui J, Corbet EF, Jin L. Combined photodynamic and low level laser therapies as an adjunct to non surgical treatment of chronic periodontitis. J Periodontal Res. 2011;46(1): 8996. doi:10.1111/j.1600-0765.2010.01316.x.

11. Page RC, Offenbacher S, Schroeder HE, Seymour GJ, Kornman KS. Advances in the pathogenesis of periodontitis: Summary of developments, clinical implications and future directions. Periodontol 2000. 1997;14:216-248. doi:10.1111/j.1600-0757.1997.tb00199.x.

12. Preshaw PM, Taylor JJ. How has research into cytokine interactions and their role in driving immune responses impacted our understanding of periodontitis? J Clin Periodontol. 2011;38 Suppl 11:60-84. doi:10.1111/j.1600051x.2010.01671.x.

13. Yao Z, Painter SL, Fanslow WC, et al. Human IL-17: A novel cytokine derived from $\mathrm{T}$ cells. J Immunol. 1995;155(12):5483-5486.

14. Aarvak T, Chabaud M, Miossec P, Natvig JB. IL-17 is produced by some proinflammatory Th1/Th0 cells but not by Th2 cells. J Immunol. 1999;162(3):1246-1251.

15. Ferretti S, Bonneau O, Dubois GR, Jones CE, Trifilieff A. IL17 , produced by lymphocytes and neutrophils, is necessary for lipopolysaccharide-induced airway neutrophilia. IL-15 as a possible trigger. J Immunol. 2003;170(4):2106-2112. doi:10.4049/jimmunol.170.4.2106.

16. Witowski J, Ksiazek K, Jorres A. Interleukin-17: a mediator of inflammatory responses. Cell Mol Life Sci. 2004;61(5):567-579.

17. Oda T, Yoshie H, Yamazaki K. Porphyromonas gingivalis antigen preferentially stimulates $\mathrm{T}$ cells to express IL-17 but not receptor activator of NF-kB ligand in vitro. Oral Microbiol Immunol. 2003;18(1):30-36.

18. Vernal R, Dutzan D, Chaparro A, Puente J, Antonieta Valanzuela M, Gamonal J. Levels of interleukin-17 in gingival crevicular fluid and in supernatants of cellular cultures of gingival tissue from patients with chronic periodontitis. J Clin Periodontol. 2005;32(4):383-389. doi:10.1111/j.1600-051x.2005.00684.x.

19. Zhao L. Zhou Y, Xu Y, Sun Y, Li L, Chen W. Effect of nonsurgical periodontal therapy on the levels of Th17/Th1/Th2 cytokines and their transcription factors in Chinese chronic periodontitis patients. J Clin Periodontol. 2011;38(6):509516. doi:10.1111/j.1600-051x.2011.01712.x.

20. Shirmohammadi A, Babaloo Z, Eskandari A, Purabbas R, Babalo A. The effects of one-stage full-mouth disinfection and Qua-drant-Wise scaling and root planing on serum levels of IL-17 and IL-1 $\beta$ and clinical parameters (A randomized controlled trial study). J Dent (Tehran). 2013;10(3):248-255.

21. Takahashi K, Azuma T, Motohira H, Kinane DF, Kitetsu S. The potential role of interleukin-17 in the immunopathology of periodontal disease. J Clin Periodontol. 2005;32(4):369374. doi:10.1111/j.1600-051x.2005.00676.x.

22. Giannopoulou C, Cappuyns I, Cancela J, Cionca N, Mombelli A. Effect of photodynamic therapy, diode laser, and deep scaling on cytokine and acute-phase protein levels in gingival crevicular fluid of residual periodontal pockets. J Periodontol. 2012;83(8):1018-1027.

23. Giannelli M, Bani D, Viti C, et al. Comparative evaluation of the effects of different photoablative laser irradiation protocols on the gingiva of periodontopathic patients. Photomed Laser Surg. 2012;30(4):222-230.

24. Moritz A, Gutknecht N, Doertbudak O, et al. Bacterial reduction in periodontal pockets through irradiation with a diode laser: A pilot study. J Clin Laser Med Surg. 1997;15(1):33-37.

25. Fontana C. R, Abernethy AD, Som S, et al. The antibacterial effect of photodynamic therapy in dental plaque-derived biofilms. J Periodontal Res. 2009;44(6):751-759.

26. Gemmell E, Seymour GJ. Immunoregulatory control of Th1/Th2 cytokine profiles in periodontal disease. Periodontol 2000. 2004;35:21-41.

27. Pradeep AR, Hadge P, Chowdhry S, Patel S, Happy D. Exploring the role of Th1 cytokines:IL-17 and IL-18 in periodontal health and disease. J Oral Sci. 2009;51(2):261266. doi:10.2334/josnusd.51.261.

28. Polansky R, Haas M, Heschl A, Wimmer G. Clinical effectiveness of photodynamic therapy in the treatment of periodontitis. J Clin Periodontol. 2009;36:575-580. doi:10.1111/j.1600-051x.2009.01412.x.

29. Chondros P, Nikolidakis D, Christodoulides N, Rossler R, Gutknecht N, Sculean A. Photodynamic therapy as adjunct to non-surgical periodontal treatment in patients on periodontal maintenance: a randomized controlled clinical trial. Lasers Med Sci. 2009;24:681-668. doi:10.1007/s10103008-0565-z.

30. Christodoulides N, Nikolidakis D, Chondros P, et al. A Photodynamic therapy as an adjunct to non-surgical periodontal treatment: a randomized, controlled clinical trial. J Periodontol. 2008;79:1638-1644.

31. de Oliveira RR, Schwartz-Filho HO, Novaes AB Jr, Taba $M$ Jr. Antimicrobial photodynamic therapy in the nonsurgical treatment of aggressive periodontitis: a preliminary randomized controlled clinical study. J Periodontol. 2007;78:965-973. doi:10.1902/jop.2007.060494.

32. Lulic M, Leiggener Gorog I, Salvi GE, Ramseier CA, Mattheos N, Lang NP. One-year outcomes of repeated adjunctive photodynamic therapy during periodontal maintenance: a proof-of-principle randomized-controlled clinical trial. J Clin Periodontol. 2009;36:661-666. doi:10.1111/j.1600-051x.2009.01432.x.

33. Yilmaz S, Kuru B, Kuru L, Noyan U, Argun D, Kadir T. Effect of gallium arsenide diode laser on human periodontal disease: a microbiological and clinical study. Lasers Surg Med. 2002;30:60-66. doi:10.1002/1sm.10010.

34. Komerik N, Wilson M, Poole S. The effect of photodynamic action on two virulence factors of gram-negative bacteria. Photochem Photobiol. 2000;72(5):676-680. 